\title{
ARTICLE
}

Cellular and Molecular Biology

\section{CREPT/RPRD1B promotes tumorigenesis through STAT3- driven gene transcription in a p300-dependent manner}

\author{
Wanli Zhai ${ }^{1,2}$, Xiongjun Ye $\mathbb{D}^{3}$, Yinyin Wang ${ }^{1}$, Yarui Feng ${ }^{1}$, Ying Wang ${ }^{1}$, Yuting Lin $^{1}{ }^{1}$, Lidan Ding ${ }^{1}$, Liu Yang ${ }^{1}$, Xuning Wang ${ }^{4}$, \\ Yanshen Kuang ${ }^{4}$, Xinyuan $\mathrm{Fu}^{5}$, Y. Eugene Chin ${ }^{6}$, Baoqing Jia ${ }^{4}$, Bingtao Zhu ${ }^{1}{ }^{1}$, Fangli Ren ${ }^{1}$ and Zhijie Chang (D)
}

BACKGROUND: Signal transducer and activator of transcription 3 (STAT3) has been shown to upregulate gene transcription during tumorigenesis. However, how STAT3 initiates transcription remains to be exploited. This study is to reveal the role of CREPT (cell cycle-related and elevated-expression protein in tumours, or RPRD1B) in promoting STAT3 transcriptional activity.

METHODS: BALB/c nude mice, CREPT overexpression or deletion cells were employed for the assay of tumour formation, chromatin immunoprecipitation, assay for transposase-accessible chromatin using sequencing.

RESULTS: We demonstrate that CREPT, a recently identified oncoprotein, enhances STAT3 transcriptional activity to promote tumorigenesis. CREPT expression is positively correlated with activation of STAT3 signalling in tumours. Deletion of CREPT led to a decrease, but overexpression of CREPT resulted in an increase, in STAT3-initiated tumour cell proliferation, colony formation and tumour growth. Mechanistically, CREPT interacts with phosphorylated STAT3 (p-STAT3) and facilitates p-STAT3 to recruit p300 to occupy at the promoters of STAT3-targeted genes. Therefore, CREPT and STAT3 coordinately facilitate p300-mediated acetylation of histone 3 (H3K18ac and H3K27ac), further augmenting RNA polymerase II recruitment. Accordingly, depletion of p300 abolished CREPT-enhanced STAT3 transcriptional activity.

CONCLUSIONS: We propose that CREPT is a co-activator of STAT3 for recruiting p300. Our study provides an alternative strategy for the therapy of cancers related to STAT3.

British Journal of Cancer (2021) 124:1437-1448; https://doi.org/10.1038/s41416-021-01269-1

\section{BACKGROUND}

Signal transducer and activator of transcription 3 (STAT3), a member of the STAT protein family, is constitutively activated in various tumours and promotes cancer progression and metastasis. ${ }^{1-5}$ STAT3 is a transcription factor that exists largely as a dimer formed by its amino-terminus domain in unstimulated cells. Upon the stimulation of cytokines or growth factors, STAT3 is phosphorylated at tyrosine 705 by tyrosine kinases (e.g. Janusactivated kinases 1, 2, and 3, or Src). After phosphorylation, the conformation of the pre-formed STAT3 dimer is stabilised by the interaction between the phosphotyrosine of one monomer and the $\mathrm{SH} 2$ domain of the other. Moreover, both unphosphorylated and phospho-Y705 STAT3 constitutively shuttles between the cytoplasmic and nuclear compartments. Reduced nuclear export due to DNA binding leads to nuclear accumulation of STAT3 in response to the cytokine. ${ }^{6-8}$ To date, it has been well documented that STAT3 regulates several genes related to cell proliferation (e.g. c-MYC and CCND1), ${ }^{9,10}$ cell survival (e.g. $B C l-X L$ and BIRC5), ${ }^{11,12}$ angiogenesis (e.g. VEGF and $\left.H G F\right)^{2,13}$ and inflammation responses (e.g. IL-6 and $I L-10) .{ }^{14-16}$ Upregulation of these genes eventually leads to cell cycle progression and resistance to apoptosis. Accordingly, normal cells with overactivated STAT3 gain the features of tumour cells. ${ }^{5,10}$ Reciprocally, elimination of STAT3 efficiently inhibits tumorigenesis and tumour cell growth in colon cancers. ${ }^{17}$ In addition, the expression and activation of STAT3 correlate with tumour grade, stage, the appearance of metastases and recurrence in breast cancers. ${ }^{18}$ To date, various STAT3 inhibitors have been developed for the therapy of different cancers. ${ }^{19-21}$ However, effective therapeutic strategies for inhibiting STAT3 remain to be further developed. ${ }^{22}$

The activity of STAT3 is precisely regulated in different cells. Both positive and negative factors have been identified to regulate the STAT3 activity. These factors include tyrosine kinases in the cytoplasm to boost the phosphorylation, ${ }^{23-25}$ phosphatases in both cytoplasm and nucleus to dephosphorylate STAT3 ${ }^{26,27}$ and enzymes to regulate STAT3 acetylation and deacetylation. ${ }^{28-30}$ Among these factors, CBP/p300 has been reported to activate STAT3 by regulating transcription initiation. CBP/p300 mediates histone modification and facilitates chromatin remodelling by enhancing histone $\mathrm{H} 3 / \mathrm{H} 4$ acetylation. ${ }^{31-34}$ The histone acetylation alteration eventually enriches the

\footnotetext{
${ }^{1}$ State Key Laboratory of Membrane Biology, School of Medicine, Tsinghua University, Beijing, China; ${ }^{2}$ Tsinghua-Peking Joint Center for Life Sciences, School of Life Science, Tsinghua University, Beijing, China; ${ }^{3}$ Urology and Lithotripsy Center, Peking University People's Hospital, Beijing, China; ${ }^{4}$ Department of General Surgery, Chinese PLA General

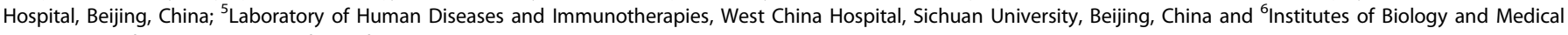
Sciences, Soochow University, Suzhou, China

Correspondence: Bingtao Zhu (bingtaozhu@163.com) or Fangli Ren (rfl@mail.tsinghua.edu.cn) or Zhijie Chang (zhijiec@tsinghua.edu.cn)

These authors contributed equally: Wanli Zhai, Xiongjun Ye
}

Received: 15 December 2019 Revised: 14 November 2020 Accepted: 5 January 2021

Published online: 3 February 2021 
recruitment of RNA polymerase II (RNAPII) to transcript STAT3targeted genes. However, how STAT3 initiates transcription remains to be exploited.

We previously identified a gene CREPT (cell cycle-related and elevated-expression protein in tumours, or RPRD1B) that is highly expressed in varieties of human tumours ${ }^{35-44}$ and also murine stem cells. ${ }^{45}$ CREPT promotes tumorigenesis through upregulation of a set of cell cycle-related genes. A mechanism study revealed that CREPT facilitates the recruitment of RNAPII to the promoter region and blocks its reading through the pol(A) signal in the termination region of the CCND1 gene. CREPT promotes a loop formation of the CCND1 gene to facilitate RNAPII redistribution to the promoter after termination ${ }^{35}$ and is found to physically interact with the C-terminal domain (CTD) of RNAPII. ${ }^{35,46,47}$ Recently, we found that p300 is a CREPTinteracting protein and CREPT increases the interaction between p300 and $\beta$-catenin to enhance the transcription of $\beta$-catenin/ TCF4 in Wnt signalling. ${ }^{48,49}$ In the present study, we demonstrate that CREPT augments the STAT3 transcriptional activity through p300-mediated histone acetylation. CREPT and p300 cooperatively promote STAT3-driven tumorigenesis. We prospect that targeting both CREPT and STAT3 will be a promising intervention for cancer therapy.

\section{METHODS}

Plasmids and reagents

Flag-CREPT, Myc-CREPT, Myc-CREPT/RPR, Myc-CREPT/CCT, FlagSTAT3, APRE (acute phase response element)-luciferase and pCDH-HA-CREPT were constructed in this laboratory. HA-p300 plasmid was a gift from Dr. Y. Eugene Chin (Institutes of Biology and Medical Sciences, Soochow University). Anti-STAT3 (c-20), anti-Myc (9E10) and anti-HA (F-7) were purchased from Santa Cruz Biotechnology (Santa Cruz). Anti-p300 (D2X6N), anti-c-MYC, anti-cyclinD1 (SP4), anti-pY705 STAT3 (D3A7), antipS727 STAT3, anti-acK685 STAT3, anti-Bcl-XL (54H6), antiH3K18ac (D8Z5H) and anti-H3K27ac (D5E4) antibodies were purchased from Cell Signaling Technology. Anti-actin (AC-15) and anti-Flag (M2) antibodies were purchased from Sigma. AntiCREPT antibody was prepared by this laboratory. ${ }^{50}$ The cytokine leukaemia inhibitory factor (LIF) was purchased from Millipore (cat. \#LIF1010). Short interfering RNAs (siRNAs) against CREPT or p300 were synthesised from GenePharma (SuZhou GenePharma Co. Ltd) with the oligo sequence information as shown in Table S1. The CRISPR/Cas9 (clustered regularly interspaced short palindromic repeats/CRISPR-associated 9)-mediated CREPT deletion plasmids were generated based on PX458M vector with guider RNAs and the sequence information was shown in Table S1.

Cell culture and transfection

HEK293T, NIH3T3 MOCK, NIH3T3 v-Src, MCF7 and SW480 cells were cultured in Dulbecco's modified Eagle's medium supplemented with $10 \%$ fetal bovine serum and penicillin/streptomycin. All the above cells were kept at $37^{\circ} \mathrm{C}$ in a $5 \% \mathrm{CO}_{2}$-containing atmosphere. Cells were transfected with plasmids as indicated using Vigofect (Vigorous Inc.), according to the manufacturer's instruction. To generate stable cell lines, 3T3 MOCK cells or 3T3 v-Src-overexpressed cells were infected by a lentivirus, which was produced by HEK293T cells transiently transfected with vectors of $\mathrm{pCDH}$ and pLL3.7 carrying different complementary DNAs. Green fluorescent protein-positive cells were selected by fluorescence-activated cell sorting. For MCF7 or SW480 CREPT deletion cell lines, PX458 vector or PX458 carrying single guide RNAs for CREPT were transiently transfected into MCF7 or SW480 cells. Several single clones were randomly picked and identified by PCR.
Luciferase assays

Indicated plasmids were co-transfected into the cells. After transfection for $24 \mathrm{~h}$, cells were stimulated with LIF $(20 \mathrm{ng} / \mathrm{ml})$ or oncostatin M (OSM) $(20 \mathrm{ng} / \mathrm{ml})$ for $8 \mathrm{~h}$. The reporter activity was examined by the Dual-Luciferase Assay System (Vigorous Inc.). Firefly luciferase activity was normalised against Renilla luciferase activity and presented as a mean \pm standard deviation.

Real-time quantitative PCR (QRT-PCR) analysis

Total RNA was extracted using TRIzol (Invitrogen). RNA was reversely transcribed using a Quantscript RT Kit (TIANGEN Biotech). QRT-PCR was performed using a Talent qPCR (quantitative polymerase chain reaction) PreMix (SYBR Green) Kit (TIANGEN Biotech) on a Roche machine using the following conditions: denature, $95^{\circ} \mathrm{C}, 5 \mathrm{~s}$; annealing, $60^{\circ} \mathrm{C}, 10 \mathrm{~s}$; and extension, $72^{\circ} \mathrm{C}, 15$ $\mathrm{s}$. The sequence of the primers used for qRT-PCRs was shown in Table S2.

Co-immunoprecipitation (co-IP)

Co-IP assay was performed as described. ${ }^{4}$ Specifically, for endogenous interaction assays, the nuclear fraction of SW480 or MCF7 cells was used for immunoprecipitation (IP).

\section{Chromatin immunoprecipitation (ChIP) assay}

In brief, $1 \times 10^{7}$ MCF7 cells in a 10-cm dish were treated with LIF for $30 \mathrm{~min}$ and fixed in 1\% formaldehyde. Chromatin was sheared into 100-500 bp fragments with sonication. The lysates prepared from these cells were incubated with indicated antibodies for IP and ChIP analyses. Eluted DNA was used as templates for qPCR amplifications. The input control was from the supernatant before precipitation. The fragment corresponding to the APRE site was amplified by PCR with the primers shown in Table S3.

\section{Immunofluorescence staining}

MCF7 cells were seeded on coverslips in a 6-well plate, incubated overnight at $37^{\circ} \mathrm{C}$ and then transfected with the indicated plasmids. Cells were treated with LIF $(20 \mathrm{ng} / \mathrm{ml})$ for $30 \mathrm{~min}$ before fixation with $4 \%$ paraformaldehyde for $20 \mathrm{~min}$ and perforated with $0.3 \%$ Triton X-100 for $10 \mathrm{~min}$. After blocking with $10 \%$ goat serum for $1 \mathrm{~h}$ at room temperature, cells were incubated with the indicated antibodies overnight at $4{ }^{\circ} \mathrm{C}$, followed by incubation with the secondary antibodies conjugated with FITC (green) or TRITC antibody (Jackson Research Laboratories) for $1 \mathrm{~h}$. Stained cells were visualised using a confocal laser scanning microscope (Olympus FV10iOil) with co-localisation of the two proteins indicated by a merged image.

\section{Experimental animals}

Twelve 5-week-old female BALB/C nude mice in an SPF grade were employed to evaluate the in vivo effect of CREPT on STAT3induced tumour growth. All mice were housed in isolated ventilated cages (maxima six mice per cage) barrier facility at Tsinghua University. The mice were maintained on a 12/12-h light/ dark cycle, $22-26^{\circ} \mathrm{C}$ with sterile pellet food and water ad libitum. The laboratory animal facility has been accredited by AAALAC (Association for Assessment and Accreditation of Laboratory Animal Care International) and the IACUC (Institutional Animal Care and Use Committee) of Tsinghua University approved all animal protocols used in this study.

Tumour formation in the nude mice

A total of $2 \times 10^{5}$ indicated cells were subcutaneously injected into the two flanks of the identical nude mice. Ten days after injection, mice were sacrificed by the euthanasia method of carbon dioxide inhalation. The volume of the tumours was measured by a ruler and the mass of the tumours was measured by an analytical balance. 
Colony formation assay

A total of $1 \times 10^{3}$ indicated cells were seeded in 6-well plates with the treatment of LIF $(20 \mathrm{ng} / \mathrm{ml})$. After 2 weeks, the cells were stained with crystal violet. Statistical results are representative of three independent experiments.

Assay for transposase-accessible chromatin using sequencing (ATAC-seq)

Briefly, 50,000 cells of each sample were used to generate DNA libraries for sequencing NextSeq500. All sequencing data were mapped on to hg19 using bowtie2. Peaks were called using MACS2.

Statistical analysis

All experiments were repeated at least three times. Data were presented as mean \pm standard deviation. Significant differences between the two groups were determined using the two-tailed Student's $t$ test.

\section{RESULTS}

CREPT expression is positively correlated with STAT3 activation in human tumours

To analyse the potential relevance of CREPT and STAT3 activation, western blot analyses were performed in human breast cancer (Fig. 1a) and colon cancer tissues (Fig. 1b). We quantified the level of the proteins and calculated the ratio of phosphorylated STAT3 ( $p$-STAT3), an activated form of STAT3 phosphorylated at $Y 705$, to total STAT3 to indicate the activation of STAT3. A statistical analysis showed that both levels of CREPT and STAT3 activation were higher in the tumour tissue than those in the paired adjacent non-tumour tissue (Fig. 1c). Furthermore, immunohistochemistry (IHC) analyses showed that CREPT and p-STAT3 were both strongly stained in the nucleus in breast and colon cancer tissues (Fig. 1d, e). To confirm the specificity of the antibody used for IHC, we stained two consecutive slices using CREPT (Fig. S1A) or p-STAT3 (Fig. S1B) antibody and an isotype control lgG. We observed a negative staining by both mouse and rabbit lgG, indicating the specificity of CREPT and p-STAT3 antibody. In addition, we used the adjacent normal tissue to compare with the tumour tissue for the correlation of CREPT and p-STAT3. Consistently, the tumour tissues exhibited higher levels of CREPT and p-STAT3 than adjacent normal tissues both in breast (Fig. 1f) and colon (Fig. 1g) cancer. The upregulation of CREPT and $\mathrm{p}$-STAT3 in colon cancer tissue compared with the normal tissue was significantly correlated $(R=0.71, p=0.038)$ (Fig. S1E). All these results suggest that the activation of STAT3 occurred simultaneously with elevated expression of CREPT in tumours.

To examine whether elevated CREPT level correlates to the expression of STAT3-downstream genes, we analysed the messenger RNA (mRNA) levels of several key genes including $c-M Y C, C C N D 1$ and $B C l-X L$ in breast and colon cancers from The Cancer Genome Atlas RNA-sequencing database. The results showed that in breast cancer, the expression of CREPT was positively correlated with $B C l-X L$ and weakly correlated with C-MYC and CCND1 at the mRNA level (Fig. S1C). However, in colon cancer, CREPT exhibited a positive correlation with those three genes (Fig. S1D). These results suggest that CREPT might participate in the regulation of STAT3-activated gene expression.

CREPT promotes tumorigenesis induced by activated STAT3 To reveal whether CREPT plays a role in the STAT3-induced tumorigenesis, a cell proliferation assay was performed in MCF7 cells under stimulation of LIF (leukaemia inhibitory factor), a cytokine that activates STAT3 ${ }^{51-55}$ (Fig. 2a). The result demonstrated that deletion of CREPT inhibited the proliferation of the cells under normal condition (WT vs. KO; Fig. 2b). Interestingly, this inhibitory role of CREPT deletion became more obvious in the presence of LIF (WT + LIF vs. KO + LIF; Fig. 2b), indicating that CREPT facilitates LIF-induced cell proliferation. In addition, a colony formation assay indicated that the colony numbers formed by MCF7 cells were significantly decreased in response to LIF when CREPT was deleted (Fig. 2c, d). Similar results were observed in SW480 cells (Fig. S2A-D). To further address the role of CREPT in STAT3-induced tumours, we transformed NIH3T3 fibroblasts using v-Src, which constitutively induces STAT3 phosphorylation, ${ }^{56}$ in the presence of stably expressed HA-CREPT (Fig. 2e). A cell proliferation assay showed that HA-CREPT promoted the cell proliferation slightly in the control cells, but dramatically in the v-Src-transformed cells (Fig. 2f). Conversely, stable depletion of CREPT by a short hairpin RNA (Fig. 2g) significantly suppressed the proliferation of v-Srctransformed cells (Fig. 2h). These results suggest that CREPT could enhance STAT3 activity in promoting cell proliferation. To examine whether CREPT could promote STAT3-induced tumour formation, v-Src-transformed 3T3 cells were subcutaneously injected into the two flanks of the identical nude mice. The results showed that the tumours formed by $v$-Src-transformed cells with overexpression of HA-CREPT were significantly larger than those formed by control cells both in mass and volume (Fig. 2i-k). Furthermore, the tumours formed by CREPT depletion cells showed a smaller volume and a less mass (Fig. 2l-n). Taken together, all the results suggest that CREPT enhances the STAT3induced tumorigenesis.

CREPT enhances STAT3 transcriptional activity and the targeted gene expression

To examine whether CREPT could regulate the STAT3 transcriptional activity, we performed a luciferase assay using the APRE reporter. ${ }^{36,57}$ Consistent with previous reports, ${ }^{51,55,58-60}$ LIF and OSM, two STAT3-activating cytokines, stimulated the STAT3 transcriptional activity, whereas overexpression of CREPT further enhanced the luciferase activity (Fig. 3a, b) in MCF7 cells. Consistently, overexpression of CREPT significantly enhanced the STAT3 transcriptional activity in SW480 (Fig. 3C, d), HEK293T (Fig. S3A) and HeLa (Fig. S3B) cells. These results suggest that CREPT could enhance the transcription activity of STAT3. To reveal the role of endogenous CREPT in STAT3-driven gene transcription, we performed a luciferase assay using specific siRNAs against CREPT. The results demonstrated that depletion of endogenous CREPT impaired the activation of luciferase in response to LIF in MCF7 (Fig. 3e, f), SW480 (Fig. 3g, h), HEK293T (Fig. S3C) and HeLa cells (Fig. S3D). These results suggest that CREPT is involved in the STAT3-driven transcriptional process. Furthermore, we found that STAT3-targeted genes including CCND1, C-MYC and BCl-XL were upregulated by overexpression of CREPT in response to LIF in MCF7 and SW480 cells at mRNA (Fig. $3 \mathrm{i}$ and Fig. S3E) and protein (Fig. $3 \mathrm{k}$ ) levels. To address the role of endogenous CREPT in the regulation of STAT3-driven genes, we depleted CREPT in MCF7 cells using siRNAs. The results showed that depletion of CREPT significantly reduced the levels of STAT3-targeted genes in response to LIF at both mRNA (Fig. $3 \mathrm{j}$ and Fig. S3F) and protein (Fig. 3l) levels. Taken together, these results suggest that CREPT is a potent regulator for the STAT3 transcriptional activity.

CREPT preferentially interacts with p-STAT3 via the RPR domain To reveal how CREPT promotes the transcriptional activity of STAT3, we addressed whether CREPT could interact with STAT3. An IP experiment using exogenously expressed Flag-STAT3 and Myc-CREPT was performed in HEK293T cells. The result showed that Myc-CREPT precipitated down Flag-STAT3 in the presence of LIF, whereas no interaction was observed without LIF (Fig. 4a), 
a

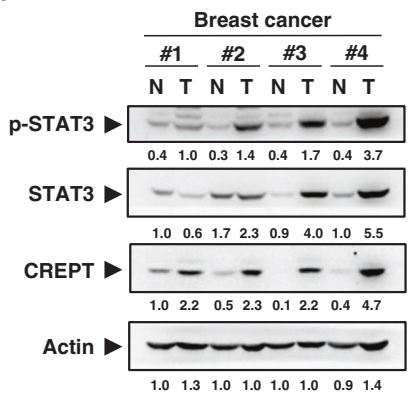

b

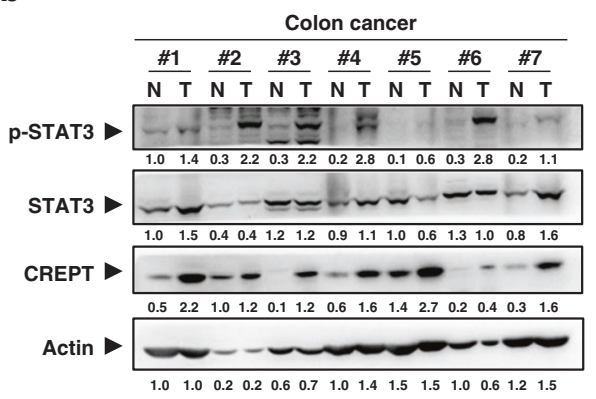

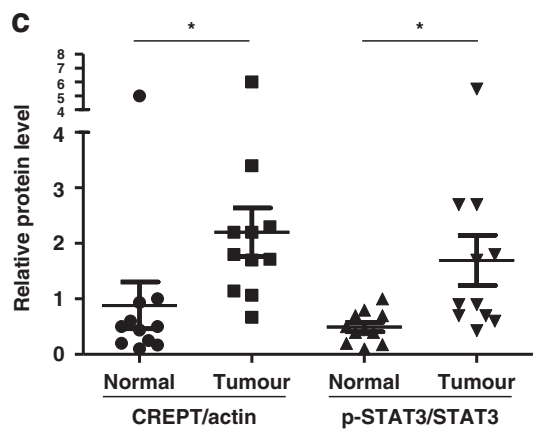

d

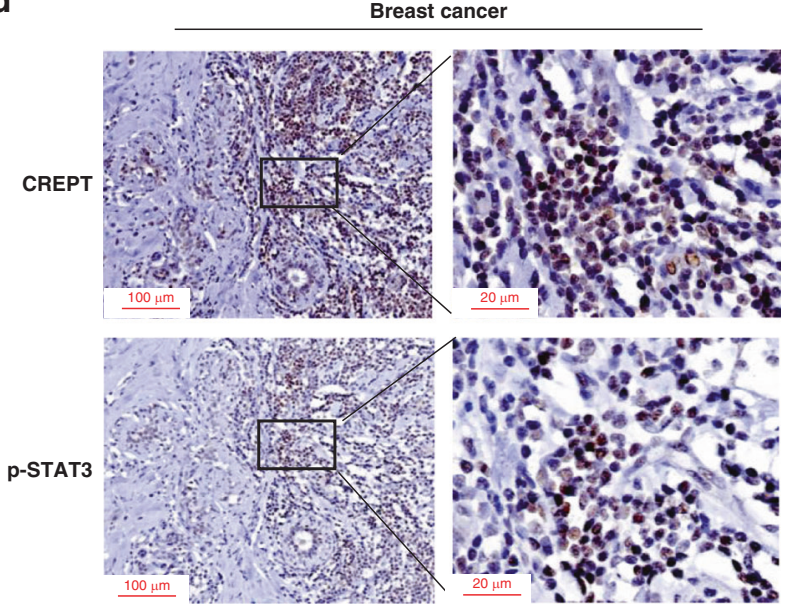

$\mathbf{f}$

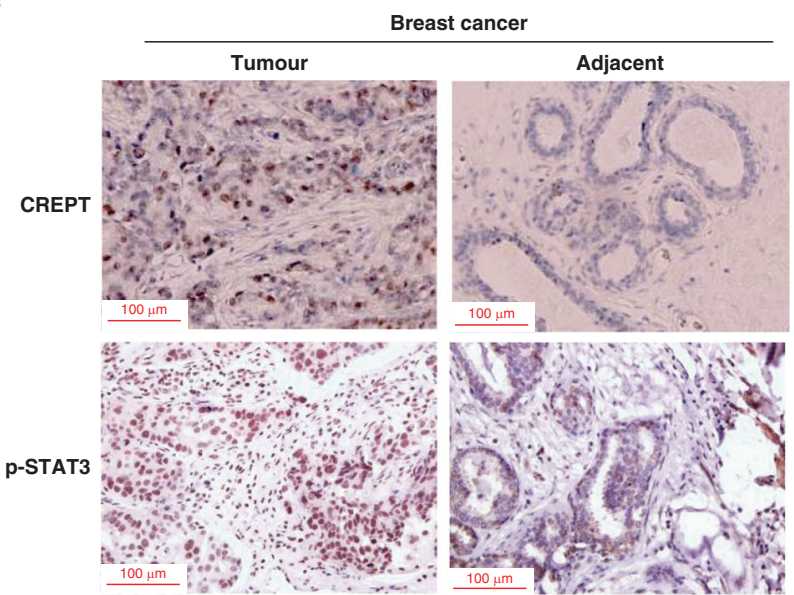

e

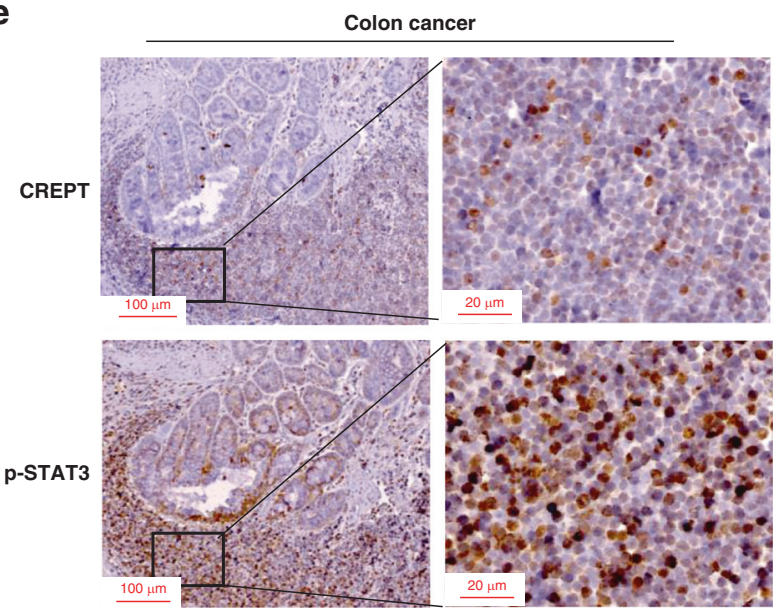

g

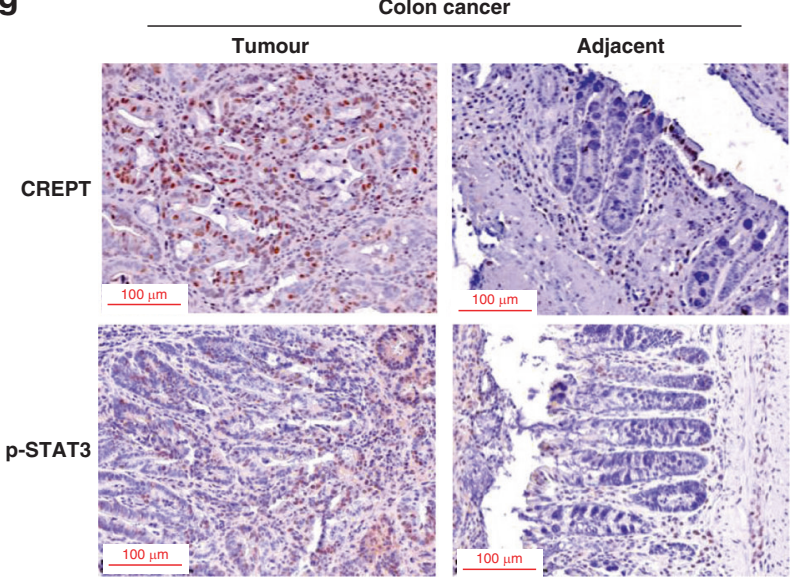

Fig. 1 CREPT expression is positively correlated with STAT3 activation in human tumours. $\mathbf{a}$, $\mathbf{b}$ The protein levels of CREPT and p-STAT3 in human breast cancers (a) and colon cancers (b). $\mathrm{N}$ refers to paired normal tissue and T refers to tumour tissue from the same patient. The bands were quantified using ImageJ and presented as a value normalised according to the moderate level of a band in each blot. c A graph presentation of CREPT/actin and p-STAT3/STAT3 in adjacent normal and tumour tissues. The grey values in a, b were used to calculate the ratio of CREPT/actin or p-STAT3/STAT3. Each sample was dotted by closed circle (normal tissue for CREPT/actin), square (tumor tissue for CREPT/actin), triangle (normal tissue for p-STAT3/STAT3) and inverted triangle (tumor tissue for p-STAT3/ STAT3), and the average was subject to a statistic analysis between normal and tumour tissues $\left({ }^{*} p<0.05\right)$. d, e Representative images for CREPT and p-STAT3 levels using immunohistochemical staining in breast (d) and colon (e) cancers with an antibody against CREPT or p-STAT3(Y705). f, $\mathbf{g}$ Representative images for CREPT and p-STAT3 in breast (f) and colon (g) tumour tissue and adjacent normal tissue.

suggesting that CREPT and STAT3 interact under the condition of LIF stimulation. Another co-IP experiment using cytoplasmic and nuclear fractions revealed that the interaction between STAT3 and CREPT occurred in the nucleus and was augmented by LIF (Fig. 4b). Since LIF induces STAT3 phosphorylation and nuclear translocation, we questioned whether the interaction of CREPT with STAT3 depends on the phosphorylation or the nuclear location of STAT3, as CREPT always locates in the nucleus. $^{35}$ We took advantage of STAT3C, a mutant that constitutively localises in the nucleus, STAT3Y705F, a mutant that lost the phosphorylation, ${ }^{61}$ and STAT3C/Y705F, a double mutant that lost the phosphorylation but remains to localise in the nucleus as a dimer. ${ }^{36}$ An IP experiment showed that STAT3Y705F failed to interact with CREPT in the presence of 


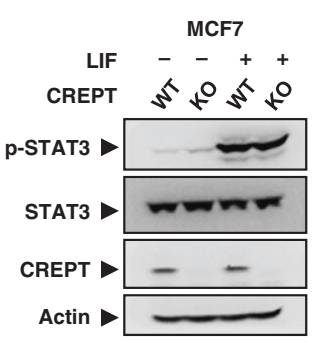

b

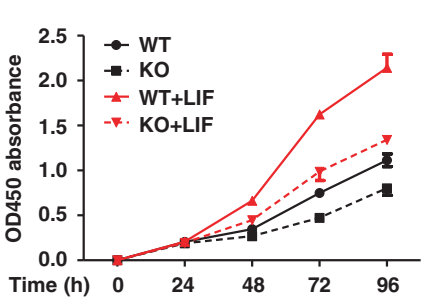

f

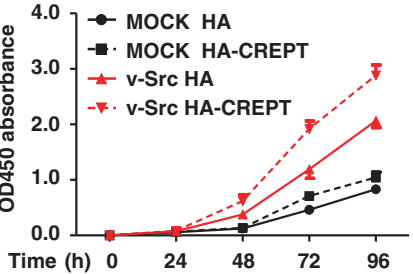

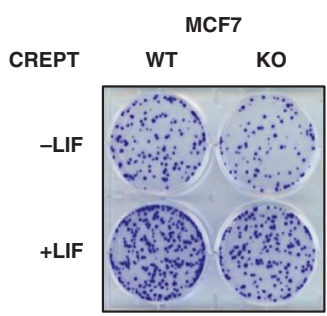

g

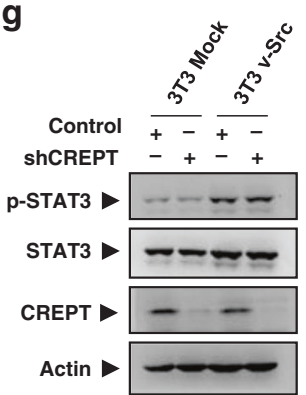

d

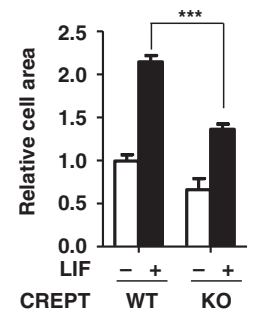

h

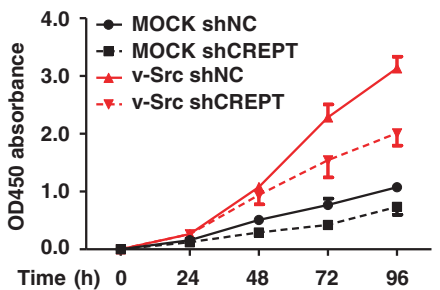

k

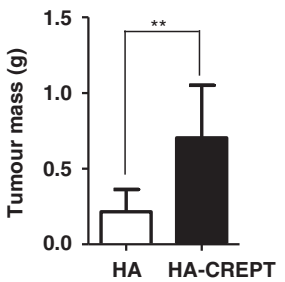

n

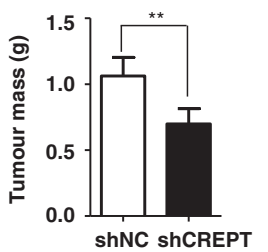

Fig. 2 CREPT promotes tumorigenesis induced by activated STAT3. a-d Deletion of CREPT reduced LIF-induced cell proliferation (a, b) or colony formation (c, d) in MCF7 cells. a Establishment of CREPT deletion and its control cell lines based on MCF7 using CRISPR/cas9 system. The cells were further treated with or without the stimulation of LIF $(20 \mathrm{ng} / \mathrm{ml})$. b A cell proliferation assay was performed using CCK8 Kit. The absorbance at $450 \mathrm{~nm}$ was used as an indicator of cell numbers. c, d Colony formation assays were performed in MCF7 cells (*** $p<0.001$ ). e Establishment of cell lines with CREPT overexpression in v-Src-transformed and control 3T3 cells. $\mathbf{f}$ Overexpression of CREPT promotes the cell growth of v-Src-transformed cells. g Establishment of cell lines with CREPT depletion in v-Src-transformed and control 3T3 cells. $\mathbf{h}$ Depletion of CREPT inhibited v-Src-transformed cell proliferation. i-k Overexpression of CREPT promotes the tumour formation from v-Srctransformed cells. Tumour formation assay was performed in nude mice $\left({ }^{* *} p<0.01\right)$. I-n Depletion of CREPT reduced the tumour formation in the $v$-Src-transformed cells $\left({ }^{* *} p<0.01\right)$.

LIF, whereas STAT3C interacted with CREPT only in the presence of LIF (Fig. 4C). Furthermore, STAT3C/Y705F, which localises in the nucleus but is not phosphorylated, failed to interact with CREPT in the presence of LIF (Fig. 4c, last lane). These results suggest that the interaction of CREPT and STAT3 depends on the phosphorylation status of STAT3.

To address whether CREPT physically binds to p-STAT3, we performed a glutathione S-transferase (GST) pull-down experiment with GST-CREPT purified from Escherichia coli and FlagSTAT3 purified from HEK293T cells under LIF stimulation. The result showed that GST-CREPT pulled down LIF-stimulated FlagSTAT3 but failed to associate with untreated Flag-STAT3 (Fig. 4d). Reciprocally, we observed that the purified GST-STAT3 protein under LIF stimulation pulled down Myc-CREPT, but untreated GST-STAT3 failed to interact with Myc-CREPT in vitro (Fig. 4e).
Obviously, p-STAT3 was detected in the LIF-stimulated GSTSTAT3 but not in the untreated GST-STAT3 (Fig. 4e). The results suggest that CREPT interacts with p-STAT3. Consistent with these in vitro results, the interaction between endogenous CREPT and STAT3 was also observed by IP experiments with an antibody against CREPT in the nuclear extracts of MCF7 (Fig. 4f) and SW480 (Fig. 4g) cells. Significantly, LIF enhanced the interaction of CREPT and STAT3 in the nucleus of both cells (Fig. 4f, g). Taken together, we conclude that CREPT interacts directly with $\mathrm{p}$-STAT3.

To analyse the detailed interaction structure of CREPT and $p$ STAT3, we generated deletion mutants with different domains of CREPT (Fig. 4h, top) and STAT3 (Fig. 4l, top). An IP experiment showed that the RPR domain, but not the CCT domain of CREPT, interacts with STAT3 (Fig. 4h). Another GST pull-down 
a

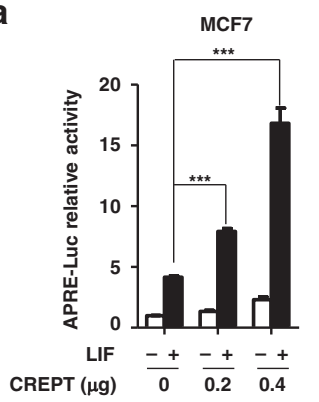

b

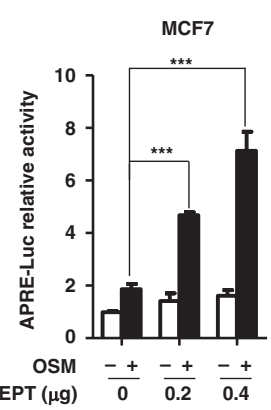

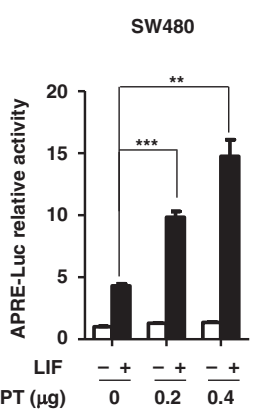

d

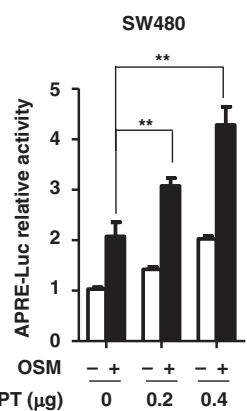

e

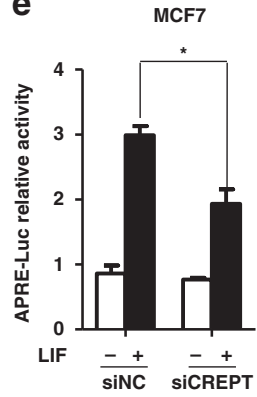

f

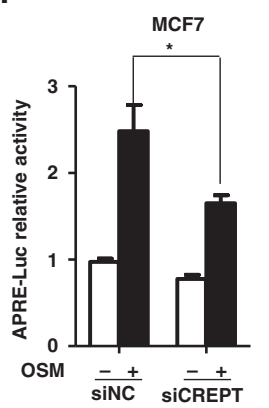

g

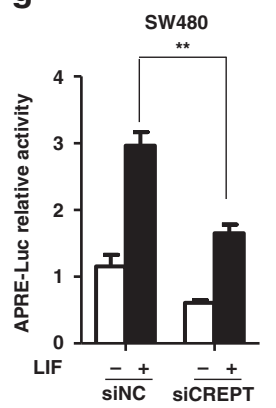

h

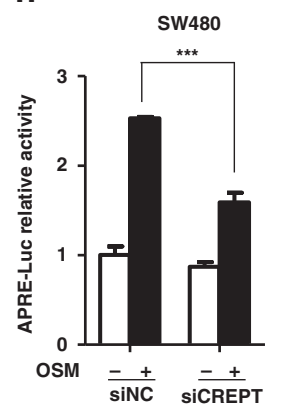

i

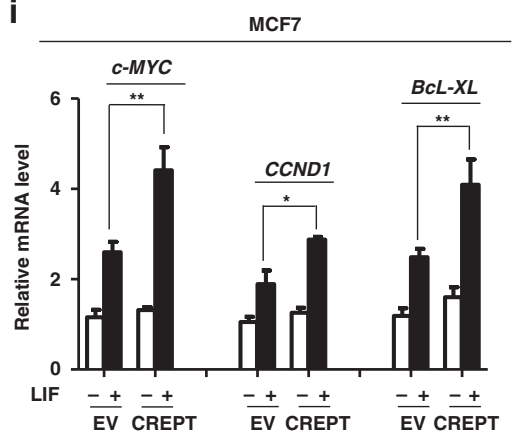

j

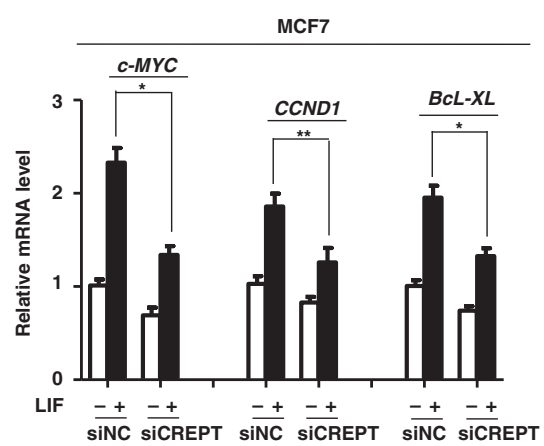

K

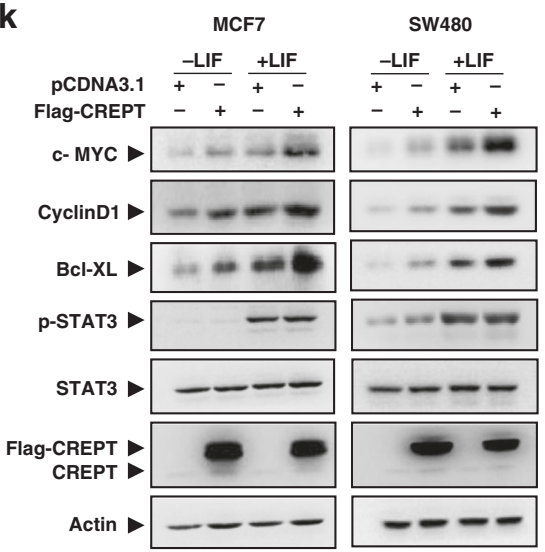

I

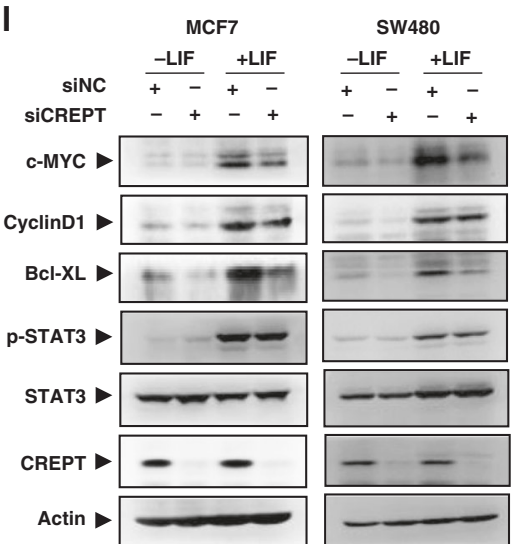

Fig. 3 CREPT enhances the STAT3 transcriptional activity and the expression of STAT3-targeted genes. a-d Overexpression of CREPT promotes the APRE-luciferase activity. Luciferase assay was performed in wild-type or CREPT transiently overexpressing cells in the absence or presence of LIF $(20 \mathrm{ng} / \mathrm{ml})\left({ }^{* *} p<0.01 ;{ }^{* *} p<0.001\right)$. e-h Depletion of CREPT using a mixture of siRNA\#1 and siRNA\#2 resulted in a decreased transcriptional activity of STAT3 $\left({ }^{*} p<0.05 ;{ }^{* *} p<0.01 ;{ }^{* *} p<0.001\right)$. i CREPT promotes the expression of STAT3-targeted genes. Flag-CREPT plasmid was transfected into MCF7 cells with or without LIF $(20 \mathrm{ng} / \mathrm{ml})$ for $4 \mathrm{~h}$. The mRNA levels of STAT3-targeted genes were examined by qRT-PCR. $\mathbf{j}$ Depletion of CREPT using a mixture of siRNA\#1 and siRNA\#2 impairs the expression of STAT3-targeted genes in response to LIF. $\mathbf{k}$ Overexpression of CREPT enhanced the protein levels of STAT3-targeted genes. Flag-CREPT plasmid was transfected into MCF7 (left) or SW480 (right) cells with or without LIF $(20 \mathrm{ng} / \mathrm{ml})$ for $12 \mathrm{~h}$. The protein levels of STAT3-targeted genes were examined by Western blots. I Depletion of CREPT decreased the protein levels of STAT3-targeted genes.

experiment using purified proteins showed that CREPT preferentially interacted with the $\mathrm{SH} 2-\mathrm{CT}$ domain and the DB-LD domain of STAT3 (Fig. 4i). Furthermore, we performed an immunostaining assay to investigate the co-localisation of CREPT with STAT3 in MCF7 cells. The results showed that CREPT co-localised with STAT3 in the nucleus after LIF treatment (Fig. 4j). Furthermore, we observed that the occupancy of CREPT on promoters of STAT3-targeted genes (c-MYC and CCND1) was enhanced by LIF stimulation and depletion of STAT3 abolished the CREPT occupancy (Fig. 4k). Our results suggest that STAT3 mediates the occupancy of CREPT at promoters of STAT3targeted genes. Collectively, all the results demonstrated that CREPT preferentially interacts with p-STAT3 to regulate STAT3 transcriptional activity.
CREPT augments histone $\mathrm{H} 3$ acetylation through enhancing p300 occupancy on the promoter of STAT3-targeted genes

To further decipher how CREPT facilitates the STAT3 transcriptional activity, we examined the possible influence of CREPT on STAT3 phosphorylation, acetylation and occupancy on the promoters of its targeted genes. Western blot analyses demonstrated that overexpression (Fig. S4A) or depletion (Fig. S4B) of CREPT had no effect on the levels of p-STAT3 (Y705), p-STAT3 (S727) and ac-STAT3 (K685), three major active forms of STAT3, 30,62 in different cell lines under LIF stimulation. In addition, a ChIP assay with primers flanking the acute phase binding element (APRE, specific for STAT3 binding) in the $c-M Y C$ or CCND1 gene demonstrated that STAT3 bound to APRE of the C-MYC or CCND1 promoter was little altered in cells with 


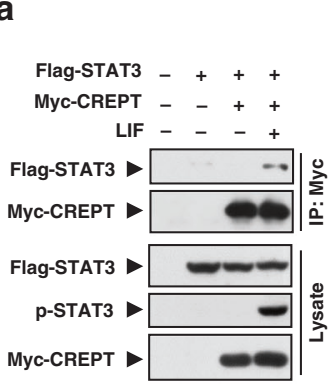

e

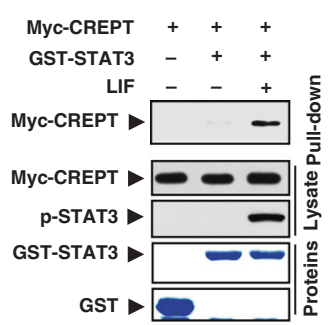

i

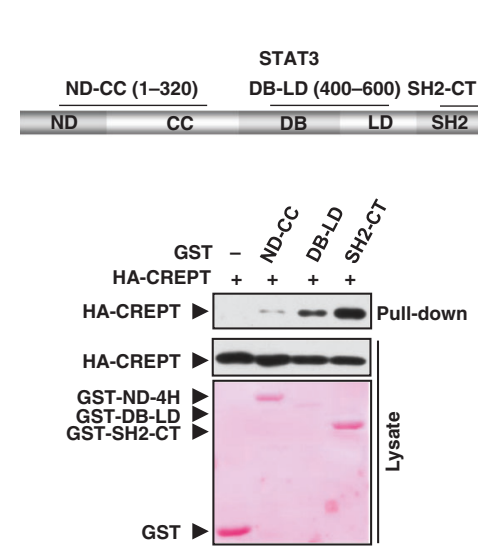

b

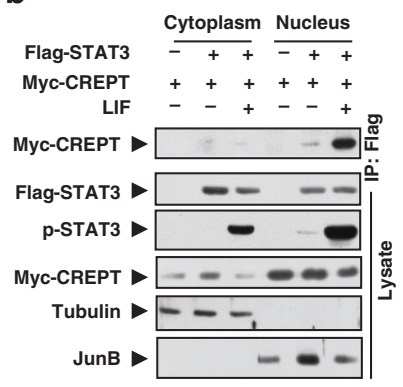

f

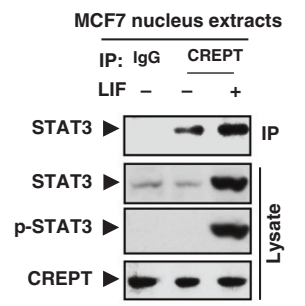

C

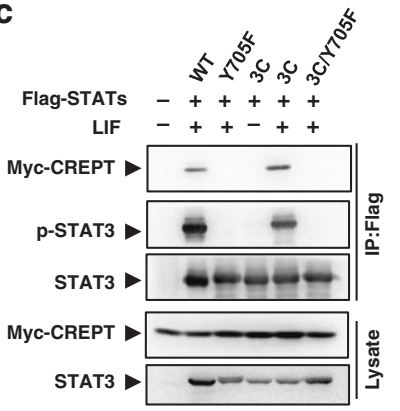

g

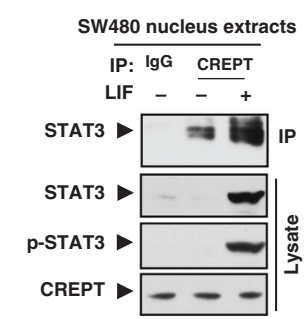

j DAPI

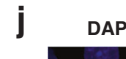

j
$\frac{\mathrm{u}}{\mathrm{u}}$

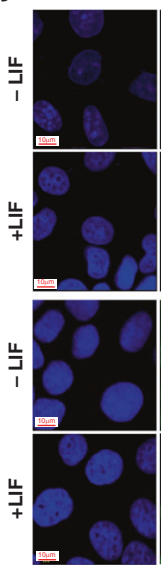

CREPT

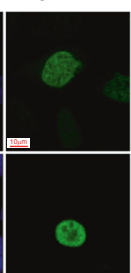

STAT3
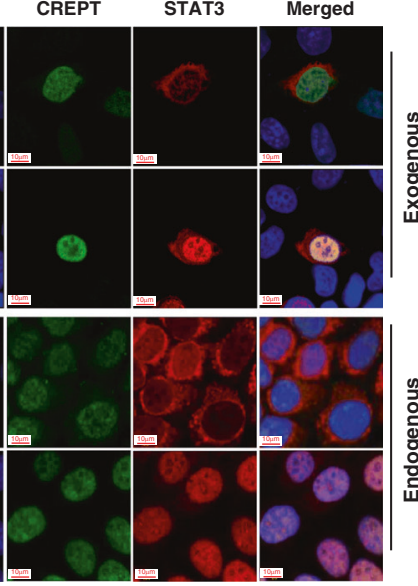

d

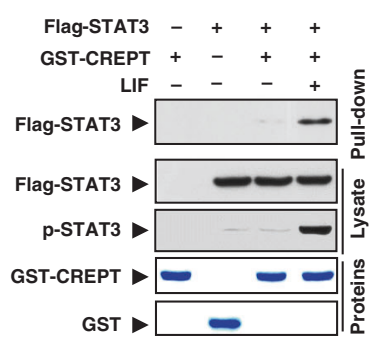

h
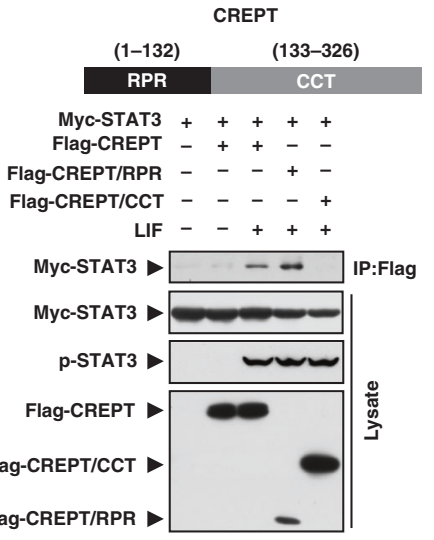

$\mathbf{k}$

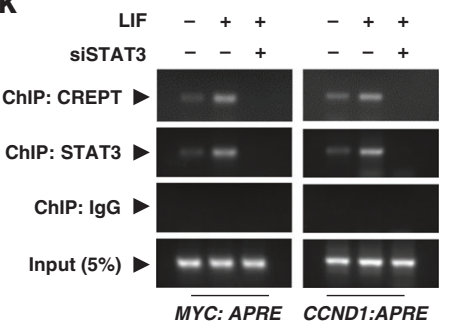

Fig. 4 CREPT preferentially interacts with p-STAT3 via its RPR domain. a Myc-CREPT interacts with Flag-STAT3 in response to LIF. b The interaction of CREPT and STAT3 occurs in the nucleus. The nuclear marker JunB and the cytoplasmic marker tubulin were used to demonstrate the purity of fractions. c Interaction of CREPT with STAT3 depends on STAT3 phosphorylation rather than its nuclear localisation. d, e CREPT interacts with STAT3 physically. A GST pull-down assay was performed with purified GST or GST-CREPT protein and Flag-STAT3 from HEK293T cell lysates (d). A reciprocal GST pull-down assay was performed with purified GST or GST-STAT3 protein and Flag-CREPT from HEK293T cell lysates (e). $\mathbf{f}, \mathbf{g}$ Endogenous interaction between CREPT and STAT3. Nuclear extracts from MCF7 (f) or SW480 (g) cells were immunoprecipitated with an anti-CREPT antibody. $\mathbf{h}$ The RPR domain, but not CCT domain of CREPT interacts with Myc-STAT3. i CREPT preferentially interacts with the SH2-CT domain of STAT3. $\mathbf{j}$ CREPT co-localises with STAT3 upon LIF treatment. MCF7 cells were treated with LIF (20 ng/ml) or a control medium for $30 \mathrm{~min}$, followed with immunostaining using an anti-CREPT antibody and an anti-STAT3 antibody. $\mathbf{k}$ CREPT and STAT3 co-occupied at the promoter region of STAT3-targeted genes. ChIP assay was performed with the CREPT or STAT3 antibody in MCF7 cells with or without the treatment of LIF $(20 \mathrm{ng} / \mathrm{ml})$ for $30 \mathrm{~min}$.

overexpression or deletion of CREPT (Fig. S4C, D). These results suggest that the enhanced transcriptional activity of STAT3 by CREPT is due to neither the increase in the STAT3 modifications nor its occupancy to the promoter region of the downstream target genes.

We further postulated that CREPT might shape the active enhancer landscape in STAT3 binding region, as enhancers are known to be the major determinants of specific gene expression. ${ }^{63}$ Therefore, the active enhancer markers H3K27ac and $\mathrm{H} 3 \mathrm{~K} 18 \mathrm{ac}$ in the promoters of STAT3-targeted genes were examined. Intriguingly, the quantitative ChIP-qPCR result showed that deletion of CREPT significantly decreased the levels of
$\mathrm{H} 3 \mathrm{~K} 27 \mathrm{ac}$ and H3K18ac at the APRE region of $c-M Y C, C C N D 1$ and $B C l-X L$ genes (Fig. 5a, b). Reciprocally, overexpression of CREPT significantly enhanced the abundance of H3K27ac and H3K18ac (Fig. S5A, B). Accordingly, an ATAC-seq result showed that loss of CREPT significantly decreased the chromatin accessibility at the promoter region of STAT3-targeted genes (Fig. $5 \mathrm{C}-\mathrm{e}$ ). These results suggest that CREPT elevated the active modifications of histone 3 flanking the STAT3 binding region. We, therefore, conclude that CREPT promotes histone modifications H3K27ac and H3K18ac to enhance the STAT3-driven gene transcription.

To reveal the mechanism how CREPT elevates the histone acetylation, we determined to investigate whether p300, a 
a

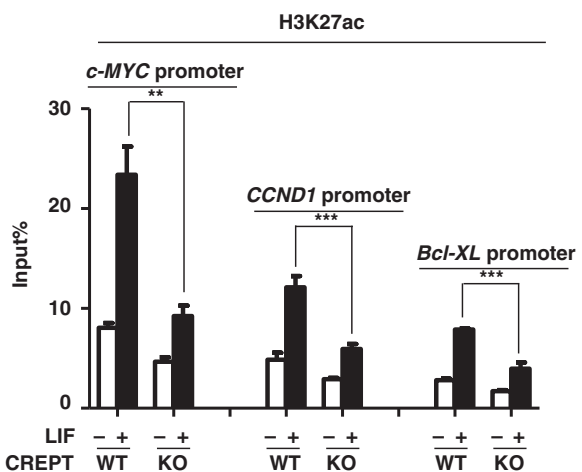

b

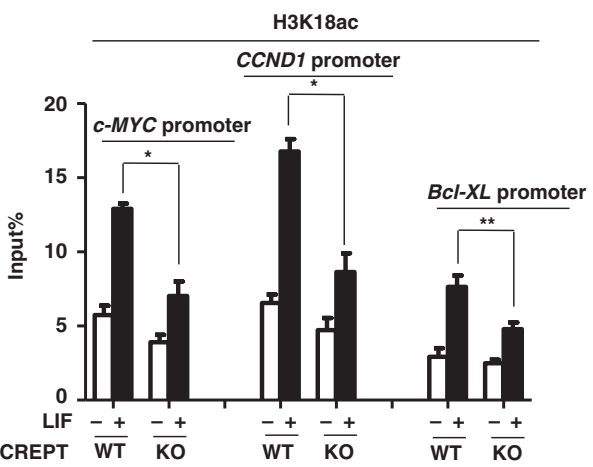

C

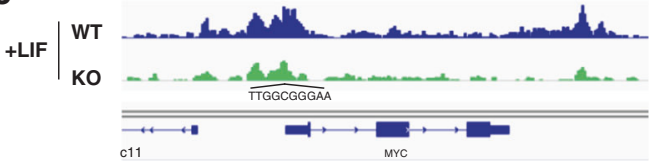

d

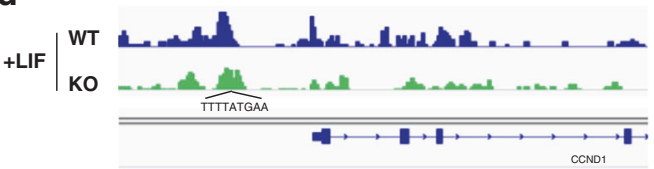

e

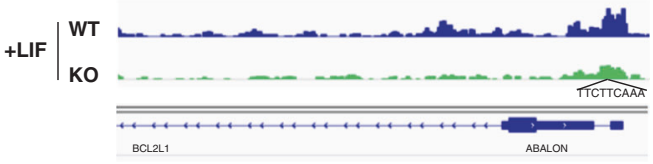

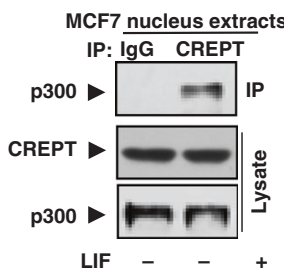

h
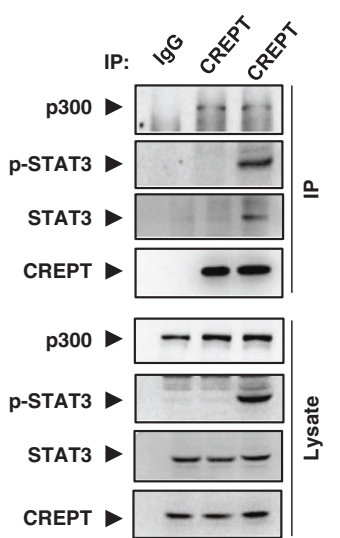

Flag-STAT3 - + + + +

SiCREPT - - + + +
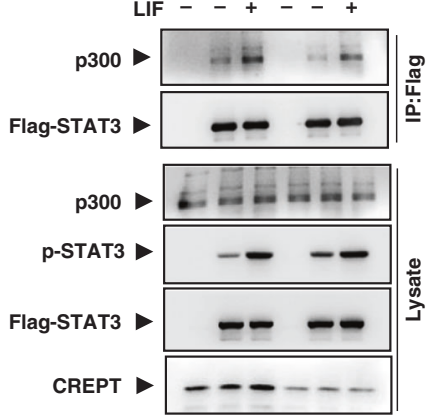

I

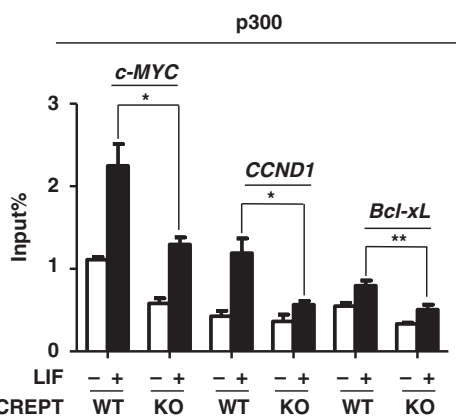

g

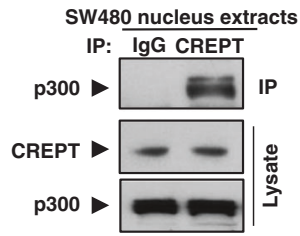

i

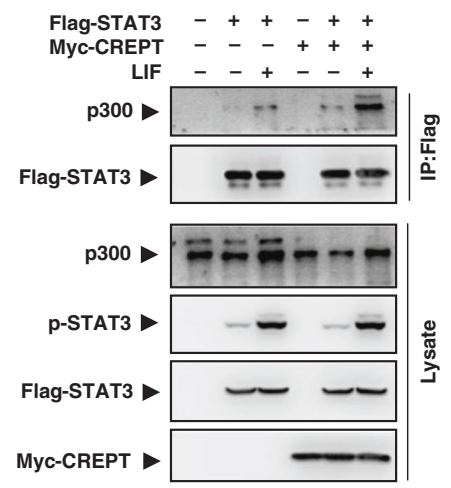

$\mathbf{k}$

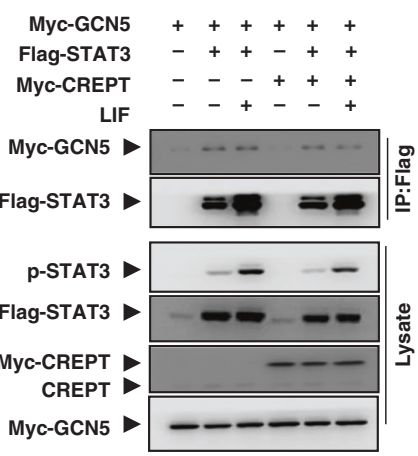

m

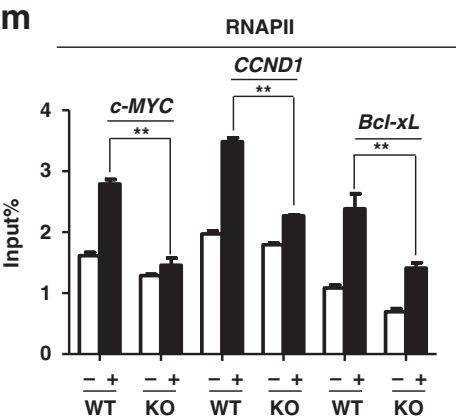

Fig. 5 CREPT augments the level of acetylated histone H3 through enhancing p300 occupancy on the promoter of STAT3-targeted genes. a, b Deletion of CREPT decreases the level of ac-H3K27 (a) or ac-H3K18 (b) on the APRE region. c-e Loss of CREPT decreases chromatin accessibility at the promoter region of STAT3. ATAC-seq peaks at the promoter regions of the STAT3-targeted genes from wild-type (blue) or CREPT depletion (green) MCF7 cells were shown. The STAT3 binding consensus sequence was labelled at the corresponding locus. $\mathbf{f}$, $\mathbf{g}$ The endogenous interaction between CREPT and p300. Nuclear extracts from MCF7 (f) or SW480 (g) cells were immunoprecipitated with an antiCREPT antibody. $\mathbf{h}$ CREPT, STAT3 and p300 formed a ternary complex in MCF7 cells. Cell lysates were precipitated by an anti-CREPT antibody. $\mathbf{i}$, j CREPT enhances the interaction of STAT3 and p300. Flag-STAT3 was co-expressed with Myc-CREPT (I) or siRNAs against endogenous CREPT (j) in MCF7 cells. $\mathbf{k}$ Overexpression of CREPT hardly affected the interaction between STAT3 and GCN5. I, $\mathbf{m}$ Deletion of CREPT attenuates p300 (I) or RNAPII $(\mathbf{m})$ occupancy on the promoters of STAT3-targeted genes. The quantity indicating the presence of p300 (I) or RNAPII (m) on APRE was normalised with respective inputs. $\left({ }^{*} p<0.05 ;{ }^{* *} p<0.01 ;{ }^{* * *} p<0.001\right)$. 
canonical histone acetyltransferase, is involved in CREPTenhanced modification of histone 3 , as p300 was isolated in the CREPT-interacting complex by our mass spectrum analysis. ${ }^{48}$ The association of endogenous CREPT with $\mathrm{p} 300$ was verified in MCF7 (Fig. 5f) and SW480 cells (Fig. 5g). Intriguingly, we observed that CREPT, p-STAT3 and p300 formed a ternary complex in MCF7 cells under LIF stimulation (Fig. 5h). To examine whether CREPT could influence the association of p300 with STAT3, a co-IP experiment was performed. The results showed that the association of p300 with STAT3 was dramatically increased in CREPT overexpression cells (Fig. 5i, lane 3 vs. 6), but was obviously decreased in CREPT depletion cells (Fig. 5j, lane 3 vs. 6). However, overexpression of CREPT failed to affect the interaction of STAT3 with GCN5, another canonical acetyltransferase (Fig. 5k). These results suggest that CREPT specifically enhances the interaction of STAT3 with p300 under LIF stimulation. Furthermore, we observed that deletion of CREPT significantly attenuated p300 occupancy to the APRE of the $C-M Y C, C C N D 1$ and $B C l-X L$ promoters (Fig. $5 \mathrm{l}$ ), suggesting that CREPT increases the association of p300 to the promoters of STAT3-targeted genes. Accordingly, the occupancy of RNAPII was also impaired in CREPT deletion cells (Fig. $5 \mathrm{~m}$ ). Taken together, all the results indicate that CREPT strengthens the p300 occupancy on the APRE region of STAT3-downstream genes and augments the histone 3 acetylation, resulting in enhanced accessibility of RNAPII.

p300 is required for CREPT-enhanced STAT3 transcriptional activity

To further verify the effect of p300 on CREPT-enhanced STAT3 transcription activation, a STAT3-specific luciferase reporter assay was performed. The results showed that overexpression of CREPT significantly enhanced the luciferase activity, but depletion of p300 abolished its effect in MCF7 (Fig. 6a) or SW480 (Fig. 6b) cells. These results suggest that $\mathrm{p} 300$ is required for CREPT in promoting STAT3-driven gene transcription. To investigate the physiological effect of p300 and CREPT on gene transcription, we examined the expression of CCND1, $C-M Y C$ and $B C l-X L$, three major STAT3-targeted genes. The results showed that CREPT failed to promote these gene expressions in the absence of p300 at both the mRNA (Fig. $6 \mathrm{c}-\mathrm{e}$ ) and protein (Fig. $6 \mathrm{f}$, g) levels. Consistently, a colony formation assay showed that CREPT promoted the cell proliferation in the presence of p300, but failed when p300 was depleted in both MCF7 (Fig. 6h, k) cells. Taken together, these data suggest that p300 is an essential factor for the CREPT-enhanced STAT3 transcriptional activity.

\section{DISCUSSION}

STAT3 has been demonstrated to play multiple roles in cell growth, cell survival and tumour immunity through activating the transcription of various genes including $c-M Y C, C C N D 1$ and $B C I-X L{ }^{9-11}$ STAT3-driven transcription requires several cofactors, such as CBP/p300, NcoA/SRC1a, and hCTR9. These factors induce modifications on histone tails, coupled with chromatin remodelling, thereby enhancing target gene expression. In the present study, we identified CREPT as a novel cofactor to regulate STAT3 transcriptional activity through p300. We proposed that CREPT bridges the interaction of STAT3 and p300 and promotes the histone acetylation for the accessibility of the chromatin (Fig. 6l). We have provided a set of evidence based on ATAC-seq analysis, gene expression patterns in the patients from The Cancer Genome Atlas database, biochemistry analyses and tumour models from mice. Our results from both human samples and mouse models suggest that CREPT regulated STAT3 activation is critical for tumorigenesis. This study extends the knowledge on underlying mechanisms of the positive regulation of STAT3 transcriptional activity by coactivators during tumorigenesis.

An important question addressed in the present study is the molecular basis for the regulation of STAT3 transcriptional activity by CREPT. The kinases of STAT3 (Src and JAK) are localised in the cytoplasm, while CREPT is localised in the nucleus. We have observed that both the canonically activated forms of STAT3, p-STAT3(Y705) ${ }^{6-8,48}$ and p-STAT3(S727), another well-known phospho-site of STAT3, essential for the maximal activation of STAT3, ${ }^{62}$ were unaffected by CREPT. Moreover, the occupancy of p-STAT3 on the promoter region was hardly affected by CREPT. These features are analogous to previous findings on the co-activator NcoA/SRC 1 a, which enhances STAT3 transcriptional activity without affecting its phosphorylation or occupancy. ${ }^{33}$ On the other hand, it is possible that CREPT expression and the activation of STAT3 could be associated in tumours. Indeed, we observed elevated CREPT is correlated with p-STAT3. The simultaneous occurrence of elevated expression of CREPT and the activation of STAT3 provides the basis for CREPT to interact with activated STAT3, enhancing its transcriptional activity through other factors. Interestingly, we observed that CREPT, p300 and p-STAT3 form a ternary complex, which enhances the enrichment of histone acetylation markers H3K18ac and H3K27ac flanking STAT3 binding region. However, CREPT fails to affect the acetylation of STAT3 proteins. This is a surprising discovery as STAT3 has been widely reported as a substrate of p300. ${ }^{30,64}$ Indeed, our experiment confirmed that LIF enhances the acetylation of STAT3. However, it appears that CREPT has no effect on LIF-induced acetylation of STAT3 (Fig. S4A, B). Because the interaction of CREPT with STAT3 is highly related to LIF stimulation but the interaction of CREPT with p300 seemed constitutive, we propose that it is p-STAT3, after its binding at the promoter, to recruit the CREPT/p300 complex to acetylate histone 3 . This mechanism is similar to the function of CREPT on the regulation Wnt/ $\beta$-catenin pathway. ${ }^{48}$ Similar mechanism was reported on the regulation of histone modification by p53 with p300 recruitment. ${ }^{65}$ Our study provides a general model for the histone modification by $\mathrm{p} 300$ through transcriptional factors, where CREPT functions as an adaptor to bridge the association of transcriptional factors, p300 and histones (Fig. 6l).

CREPT has no typical DNA-binding domain, but indeed occupies on the promoter region of selected genes. ${ }^{35,39,49}$ Our results showed that LIF stimulation increases the occupancy of CREPT on STAT3-targeted gene promoters. We propose that it is STAT3 that selects the DNA sequences at the promoter to recruit CREPT occupancy. We speculate that other transcriptional factors might also recruit CREPT to specific transcriptional sites. As CREPT also interests with RNAPII, ${ }^{35,46,66-68}$ we consider that sequence-specific transcriptional factors recruit CREPT, which mediates the recruitment of RNAPII at the transcription initiation. In this study, we confirmed our hypothesis by providing an enhanced complex formation of CREPT/p300 to promote RNAPII occupancy directed by STAT3 into specific gene promoters such as CCND1, c-MYC and BCl-XL. Furthermore, we will identify the elaborate binding site for STAT3 in CREPT by truncation-mediated immunoprecipitation assay. A smallmolecule inhibitor designed by the binding site could disturb the interaction between CREPT and STAT3, thus interfering the development of STAT3-related tumours. In addition, as the advance of PROTAC in targeting CREPT, 69 we expect that targeting the interaction of CREPT and STAT3, for instance, the $\mathrm{SH} 2-\mathrm{CT}$ fragment, will be more efficient to inhibit tumour growth.

In conclusion, we propose that STAT3 recruits CREPT to associate with p300 to acetylate histone 3 at the promoters of STAT3-targeted genes. This is critical for the oncogene 
a

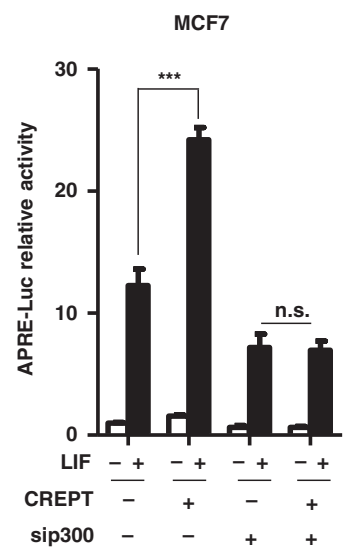

e

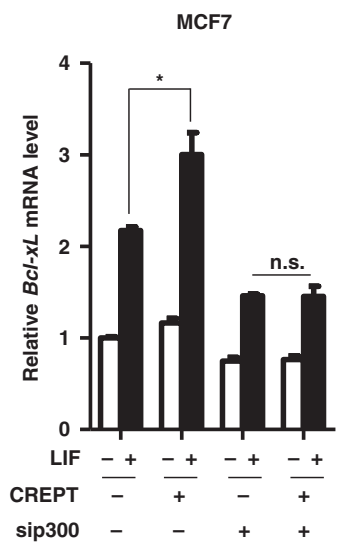

j

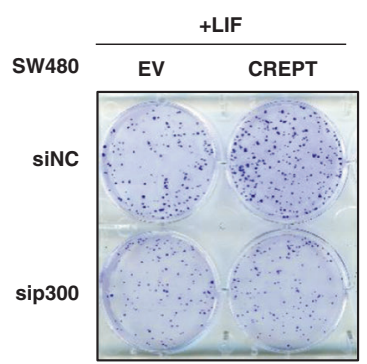

k

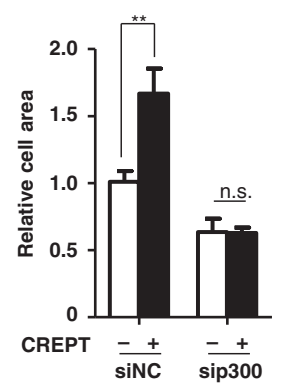

b

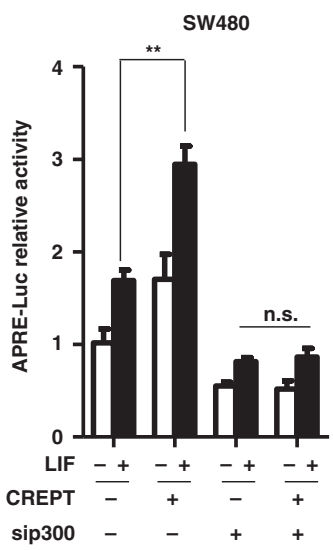

f

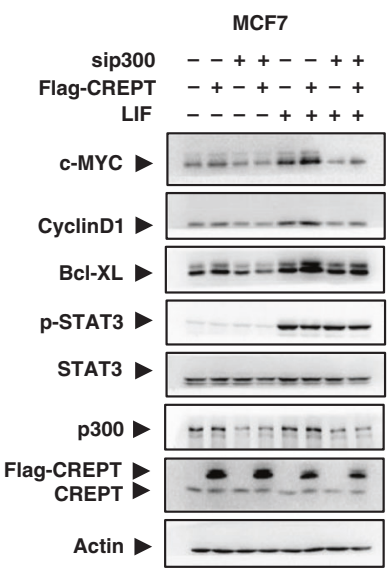

C

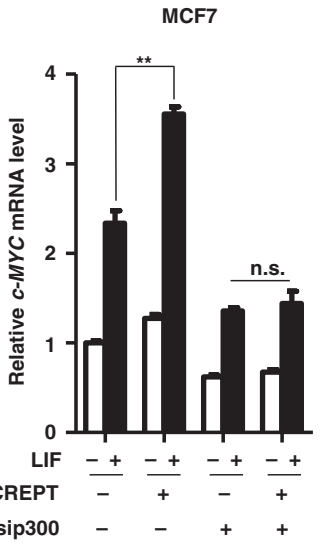

$\mathbf{g}$

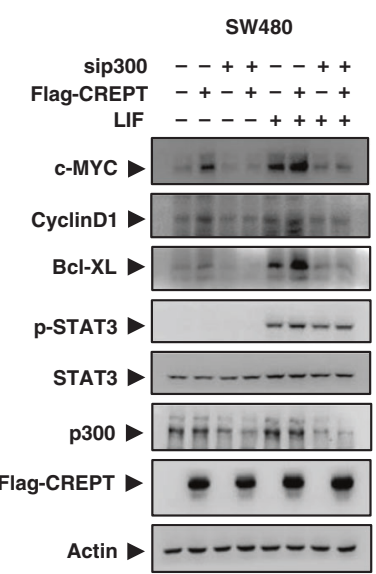

I

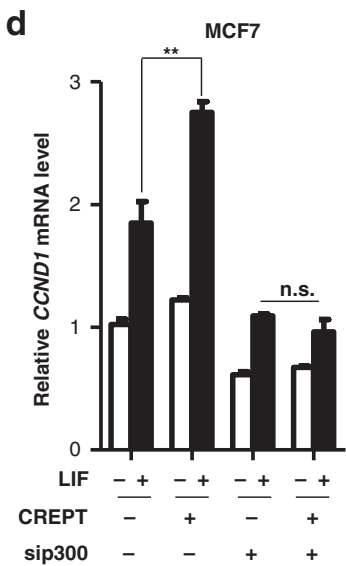

h

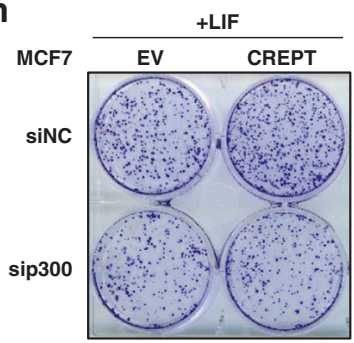

i

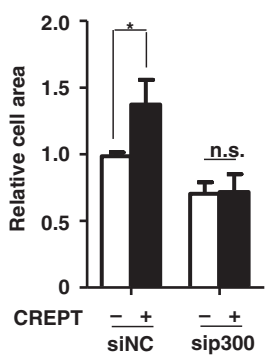

Fig. 6 p300 is required for CREPT-enhanced STAT3 transcriptional activity. a, b Depletion of p300 impaired the enhanced effect of CREPT on STAT3 transcriptional activity. $\mathbf{c}-\mathbf{g}$ Depletion of p300 impaired the STAT3-targeted gene expression promoted by CREPT both at mRNA (c-e) and protein $(\mathbf{f}, \mathbf{g})$ levels examined by QRT-PCR and western blot. $\mathbf{h}-\mathbf{k}$ CREPT promotes colony formation dependent on p300. Colony formation assays were performed in MCF7 $(\mathbf{h}, \mathbf{i})$ or SW480 (j, $\mathbf{k})$ cells. A representative colony is shown in $(\mathbf{h}, \mathbf{J})$ and the quantitative numbers are shown in $(\mathbf{i}, \mathbf{k})$. I A model indicating that highly expressed CREPT in tumour cells facilitates the recruitment of p300 to the promoter of STAT3-targeted genes $\left({ }^{*} p<0.05 ;{ }^{* *} p<0.01 ;{ }^{* *} p<0.001 ;\right.$ n.s., no statistical difference). 
transcription during tumorigenesis. We prospect that targeting CREPT can be used to develop synthetic inhibitors for STAT3related tumours.

\section{ACKNOWLEDGEMENTS}

We thank Dr. Boan Li (School of Life Sciences, Xiamen University) for kindly providing the plasmid Myc-GCN5.

\section{AUTHOR CONTRIBUTIONS}

W.Z. and X.Y. performed most of the experiments, analysed the data and wrote the paper. Z.C., F.R., B.Z. and Yinyin.W. supervised the project, critically reviewed the results and revised the paper. Y.F., Ying.W., Y.L., L.D. and L.Y. were involved in performing the experiments of ChIP-QPCR, ATAC-seq and tumour formation. X.W. and Y.K. contributed to the bioinformatics analyses. X.F., Y.E.C. and B.J. designed some of the IHC, ChIP experiments and interpreted the results.

\section{ADDITIONAL INFORMATION}

Ethics approval and consent to participate This study was performed in accordance with the Declaration of Helsinki. Archived human specimens were collected under the Ethical Committee of Tsinghua University (IRB-00008273). Written informed consent was obtained from all patients before study entry. Animal experiments were approved by the Institutional Animal Care and Use Committee in Tsinghua University (18-CZJ2).

Data availability Raw data and materials generated during the present study are available from the corresponding author upon reasonable request.

Competing interests The authors declare no competing interests.

Funding information This work was supported by grants from the Chinese National Major Scientific Research Programme (2016YFA0500301) and the National Natural Science Foundation of China (81830092, 81572728, 81572729, 81872249 and 81224487)

Supplementary information The online version contains supplementary material available at https://doi.org/10.1038/s41416-021-01269-1.

Note This work is published under the standard license to publish agreement. After 12 months the work will become freely available and the license terms will switch to a Creative Commons Attribution 4.0 International (CC BY 4.0).

Publisher's note Springer Nature remains neutral with regard to jurisdictional claims in published maps and institutional affiliations.

\section{REFERENCES}

1. Teng, Y., Ross, J. L. \& Cowell, J. K. The involvement of JAK-STAT3 in cell motility, invasion, and metastasis. JAK-STAT 3, e28086 (2014).

2. Wei, D., Le, X., Zheng, L., Wang, L., Frey, J. A., Gao, A. C. et al. Stat3 activation regulates the expression of vascular endothelial growth factor and human pancreatic cancer angiogenesis and metastasis. Oncogene 22, 319-329 (2003).

3. Garcia, R. \& Jove, R. Activation of STAT transcription factors in oncogenic tyrosine kinase signaling. J. Biomed. Sci. 5, 79-85 (1998).

4. Ahmad, R., Rajabi, H., Kosugi, M., Joshi, M. D., Alam, M., Vasir, B. et al. MUC1-C oncoprotein promotes STAT3 activation in an autoinductive regulatory loop. Sci. Signal. 4, ra9 (2011).

5. Yu, C. L., Meyer, D. J., Campbell, G. S., Larner, A. C., Carter-Su, C., Schwartz, J. et al. Enhanced DNA-binding activity of a Stat3-related protein in cells transformed by the Src oncoprotein. Science 269, 81-83 (1995).

6. Darnell, J. E. Jr. STATs and gene regulation. Science 277, 1630-1635 (1997).

7. Stark, G. R., Kerr, I. M., Williams, B. R., Silverman, R. H. \& Schreiber, R. D. How cells respond to interferons. Annu. Rev. Biochem. 67, 227-264 (1998).

8. Turkson, J., Bowman, T., Garcia, R., Caldenhoven, E., De Groot, R. P. \& Jove, R. Stat3 activation by Src induces specific gene regulation and is required for cell transformation. Mol. Cell. Biol. 18, 2545-2552 (1998).

9. Leslie, K., Lang, C., Devgan, G., Azare, J., Berishaj, M., Gerald, W. et al. Cyclin D1 is transcriptionally regulated by and required for transformation by activated signal transducer and activator of transcription 3. Cancer Res. 66, 2544-2552 (2006).
10. Bowman, T., Broome, M. A., Sinibaldi, D., Wharton, W., Pledger, W. J., Sedivy, J. M. et al. Stat3-mediated Myc expression is required for Src transformation and PDGF-induced mitogenesis. Proc. Natl Acad. Sci. USA 98, 7319-7324 (2001).

11. Shen, Y., Devgan, G., Darnell, J. E. Jr. \& Bromberg, J. F. Constitutively activated Stat3 protects fibroblasts from serum withdrawal and UV-induced apoptosis and antagonizes the proapoptotic effects of activated Stat1. Proc. Natl Acad. Sci. USA 98, 1543-1548 (2001).

12. Gritsko, T., Williams, A., Turkson, J., Kaneko, S., Bowman, T., Huang, M. et al. Persistent activation of stat3 signaling induces survivin gene expression and confers resistance to apoptosis in human breast cancer cells. Clin. Cancer Res. 12, 11-19 (2006).

13. Yu, H., Kortylewski, M. \& Pardoll, D. Crosstalk between cancer and immune cells: role of STAT3 in the tumour microenvironment. Nat. Rev. Immunol. 7, 41-51 (2007).

14. Sumimoto, H., Imabayashi, F., Iwata, T. \& Kawakami, Y. The BRAF-MAPK signaling pathway is essential for cancer-immune evasion in human melanoma cells. J. Exp. Med. 203, 1651-1656 (2006).

15. Herbeuval, J. P., Lelievre, E., Lambert, C., Dy, M. \& Genin, C. Recruitment of STAT3 for production of IL-10 by colon carcinoma cells induced by macrophage-derived IL-6. J. Immunol. 172, 4630-4636 (2004).

16. Kinjyo, I., Inoue, H., Hamano, S., Fukuyama, S., Yoshimura, T., Koga, K. et al. Loss of SOCS3 in T helper cells resulted in reduced immune responses and hyperproduction of interleukin 10 and transforming growth factor-beta 1. J. Exp. Med. 203, 1021-1031 (2006).

17. Grivennikov, S., Karin, E., Terzic, J., Mucida, D., Yu, G. Y., Vallabhapurapu, S. et al. IL6 and Stat 3 are required for survival of intestinal epithelial cells and development of colitis-associated cancer. Cancer Cell 15, 103-113 (2009).

18. Hsieh, F. C., Cheng, G. \& Lin, J. Evaluation of potential Stat3-regulated genes in human breast cancer. Biochem. Biophys. Res. Commun. 335, 292-299 (2005).

19. Zhang, X., Yue, P., Page, B. D., Li, T., Zhao, W., Namanja, A. T. et al. Orally bioavailable small-molecule inhibitor of transcription factor Stat3 regresses human breast and lung cancer xenografts. Proc. Natl Acad. Sci. USA 109, 9623-9628 (2012).

20. Huang, W., Liu, Y., Wang, J., Yuan, X., Jin, H. W., Zhang, L. R. et al. Small-molecule compounds targeting the STAT3 DNA-binding domain suppress survival of cisplatin-resistant human ovarian cancer cells by inducing apoptosis. Eur. J. Med. Chem. 157, 887-897 (2018).

21. Huang, W., Dong, Z., Wang, F., Peng, H., Liu, J. Y. \& Zhang, J. T. A small molecule compound targeting STAT3 DNA-binding domain inhibits cancer cell proliferation, migration, and invasion. ACS Chem. Biol. 9, 1188-1196 (2014).

22. Yu, H., Lee, H., Herrmann, A., Buettner, R. \& Jove, R. Revisiting STAT3 signalling in cancer: new and unexpected biological functions. Nat. Rev. Cancer 14, 736-746 (2014).

23. Martin, G. S. The hunting of the Src. Nat. Rev. Mol. Cell Biol. 2, 467-475 (2001).

24. Heinrich, P. C., Behrmann, I., Haan, S., Hermanns, H. M., Muller-Newen, G. \& Schaper, F. Principles of interleukin (IL)-6-type cytokine signalling and its regulation. Biochem. J. 374, 1-20 (2003).

25. Darnell, J. E. Jr. Studies of IFN-induced transcriptional activation uncover the JakStat pathway. J. Interferon Cytokine Res. 18, 549-554 (1998).

26. Yagil, Z., Nechushtan, H., Kay, G., Yang, C. M., Kemeny, D. M. \& Razin, E. The enigma of the role of protein inhibitor of activated STAT3 (PIAS3) in the immune response. Trends Immunol. 31, 199-204 (2010).

27. Yoshimura, A., Naka, T. \& Kubo, M. SOCS proteins, cytokine signalling and immune regulation. Nat. Rev. Immunol. 7, 454-465 (2007).

28. Ni, L., Wang, L., Yao, C., Ni, Z., Liu, F., Gong, C. et al. The histone deacetylase inhibitor valproic acid inhibits NKG2D expression in natural killer cells through suppression of STAT3 and HDAC3. Sci. Rep. 7, 45266 (2017).

29. Ray, S., Sherman, C. T., Lu, M. \& Brasier, A. R. Angiotensinogen gene expression is dependent on signal transducer and activator of transcription 3-mediated p300/cAMP response element binding protein-binding protein coactivator recruitment and histone acetyltransferase activity. Mol. Endocrinol. 16, 824-836 (2002).

30. Yuan, Z. L., Guan, Y. J., Chatterjee, D. \& Chin, Y. E. Stat3 dimerization regulated by reversible acetylation of a single lysine residue. Science 307, 269-273 (2005).

31. Hou, T., Ray, S., Lee, C. \& Brasier, A. R. The STAT3 NH2-terminal domain stabilizes enhanceosome assembly by interacting with the p300 bromodomain. J. Biol. Chem. 283, 30725-30734 (2008).

32. Lerner, L., Henriksen, M. A., Zhang, X. \& Darnell, J. E. Jr. STAT3-dependent enhanceosome assembly and disassembly: synergy with GR for full transcriptional increase of the alpha 2-macroglobulin gene. Genes Dev. 17, 2564-2577 (2003).

33. Cheng, P. Y., Lin, Y. P., Chen, Y. L., Lee, Y. C., Tai, C. C., Wang, Y. T. et al. Interplay between SIN3A and STAT3 mediates chromatin conformational changes and GFAP expression during cellular differentiation. PLOS ONE 6, e22018 (2011). 
34. Giraud, S., Bienvenu, F., Avril, S., Gascan, H., Heery, D. M. \& Coqueret, O. Functional interaction of STAT3 transcription factor with the coactivator NcoA/SRC1a. J. Biol. Chem. 277, 8004-8011 (2002).

35. Lu, D., Wu, Y., Wang, Y., Ren, F., Wang, D., Su, F. et al. CREPT accelerates tumorigenesis by regulating the transcription of cell-cycle-related genes. Cancer Cell 21, 92-104 (2012)

36. Wang, Y., Ning, H., Ren, F., Zhang, Y., Rong, Y., Wang, Y. et al. GdX/UBL4A specifically stabilizes the TC45/STAT3 association and promotes dephosphorylation of STAT3 to repress tumorigenesis. Mol. Cell 53, 752-765 (2014).

37. Ma, J., Ren, Y., Zhang, L., Kong, X., Wang, T., Shi, Y. et al. Knocking-down of CREPT prohibits the progression of oral squamous cell carcinoma and suppresses cyclin D1 and c-Myc expression. PLOS ONE 12, e0174309 (2017).

38. Zheng, G., Li, W., Zuo, B., Guo, Z., Xi, W., Wei, M. et al. High expression of CREPT promotes tumor growth and is correlated with poor prognosis in colorectal cancer. Biochem. Biophys. Res. Commun. 480, 436-442 (2016).

39. Ding, L., Yang, L., He, Y., Zhu, B., Ren, F., Fan, X. et al. CREPT/RPRD1B associates with Aurora B to regulate Cyclin B1 expression for accelerating the G2/M transition in gastric cancer. Cell Death Dis. 9, 1172 (2018).

40. She, Y., Liang, J., Chen, L., Qiu, Y., Liu, N., Zhao, X. et al. CREPT expression correlates with poor prognosis in patients with retroperitoneal leiomyosarcoma. Int. J. Clin. Exp. Pathol. 7, 6596-6605 (2014).

41. Liu, H., Seynhaeve, A. L. B., Brouwer, R. W. W., van IJcken, W. F. J., Yang, L., Wang, Y. et al. CREPT promotes melanoma progression through accelerated proliferation and enhanced migration by RhoA-mediated actin filaments and focal adhesion formation. Cancers 12, https://doi.org/10.3390/cancers12010033 (2019).

42. Komor, M. A., de Wit, M., van den Berg, J., Martens de Kemp, S. R., Delis-van Diemen, P. M., Bolijn, A. S. et al. Molecular characterization of colorectal adenomas reveals POFUT1 as a candidate driver of tumor progression. Int. J. Cancer 146, 1979-1992 (2020).

43. Yang, G., Wang, Y., Xiao, J., Zhao, F., Qiu, J., Liu, Y. et al. CREPT serves as a biomarker of poor survival in pancreatic ductal adenocarcinoma. Cell. Oncol. https://doi.org/10.1007/s13402-020-00569-7 (2020).

44. Li, M. M. D. \& Chang, Z. Current understanding of CREPT and p15RS, carboxyterminal domain (CTD)-interacting proteins, in human cancers. Oncogene https:// doi.org/10.1038/s41388-020-01544-0 (in the press).

45. Yang, L. Y. H., Chu, Y., Song, Y., Ding, L., Zhu, B. et al. CREPT is required for murine stem cell maintenance during intestinal regeneration. Nat. Commun. 12, 270 https://doi.org/10.1038/s41467-020-20636-9 (2021).

46. Ni, Z., Olsen, J. B., Guo, X., Zhong, G., Ruan, E. D., Marcon, E. et al. Control of the RNA polymerase II phosphorylation state in promoter regions by CTD interaction domain-containing proteins RPRD1A and RPRD1B. Transcription 2, 237-242 (2011).

47. Patidar, P. L., Motea, E. A., Fattah, F. J., Zhou, Y., Morales, J. C., Xie, Y. et al. The Kub5-Hera/RPRD1B interactome: a novel role in preserving genetic stability by regulating DNA mismatch repair. Nucleic Acids Res. 44, 1718-1731 (2016).

48. Zhang, Y., Wang, S., Kang, W., Liu, C., Dong, Y., Ren, F. et al. CREPT facilitates colorectal cancer growth through inducing Wnt/beta-catenin pathway by enhancing p300-mediated beta-catenin acetylation. Oncogene 37, 3485-3500 (2018).

49. Zhang, Y., Liu, C., Duan, X., Ren, F., Li, S., Jin, Z. et al. CREPT/RPRD1B, a recently identified novel protein highly expressed in tumors, enhances the beta-catenin. TCF4 transcriptional activity in response to Wnt signaling. J. Biol. Chem. 289, 22589-22599 (2014)

50. Ren, F., Wang, R., Zhang, Y., Liu, C., Wang, Y., Hu, J. et al. Characterization of a monoclonal antibody against CREPT, a novel protein highly expressed in tumors. Monoclon. Antib. Immunodiagn. Immunother. 33, 401-408 (2014).

51. Estrov, Z., Samal, B., Lapushin, R., Kellokumpu-Lehtinen, P., Sahin, A. A., Kurzrock, $R$. et al. Leukemia inhibitory factor binds to human breast cancer cells and stimulates their proliferation. J. Interferon Cytokine Res. 15, 905-913 (1995).
52. Kellokumpu-Lehtinen, P., Talpaz, M., Harris, D., Van, Q., Kurzrock, R. \& Estrov, Z. Leukemia-inhibitory factor stimulates breast, kidney and prostate cancer cell proliferation by paracrine and autocrine pathways. Int. J. Cancer 66, 515-519 (1996).

53. Quaglino, A., Schere-Levy, C., Romorini, L., Meiss, R. P. \& Kordon, E. C. Mouse mammary tumors display Stat3 activation dependent on leukemia inhibitory factor signaling. Breast Cancer Res. 9, R69 (2007).

54. Li, X., Yang, Q., Yu, H., Wu, L., Zhao, Y., Zhang, C. et al. LIF promotes tumorigenesis and metastasis of breast cancer through the AKT-mTOR pathway. Oncotarget $\mathbf{5}$, 788-801 (2014)

55. Yu, H., Yue, X., Zhao, Y., Li, X., Wu, L., Zhang, C. et al. LIF negatively regulates tumour-suppressor p53 through Stat3/ID1/MDM2 in colorectal cancers. Nat. Commun. 5, 5218 (2014).

56. Cao, X., Tay, A., Guy, G. R. \& Tan, Y. H. Activation and association of Stat3 with Src in v-Src-transformed cell lines. Mol. Cell. Biol. 16, 1595-1603 (1996).

57. Rong, Y., Cheng, L., Ning, H., Zou, J., Zhang, Y., Xu, F. et al. Wilms' tumor 1 and signal transducers and activators of transcription 3 synergistically promote cell proliferation: a possible mechanism in sporadic Wilms' tumor. Cancer Res. 66, 8049-8057 (2006)

58. West, N. R. \& Watson, P. H. S100A7 (psoriasin) is induced by the proinflammatory cytokines oncostatin-M and interleukin-6 in human breast cancer. Oncogene 29, 2083-2092 (2010).

59. Snyder, M., Huang, X. Y. \& Zhang, J. J. Signal transducers and activators of transcription 3 (STAT3) directly regulates cytokine-induced fascin expression and is required for breast cancer cell migration. J. Biol. Chem. 286, 38886-38893 (2011).

60. Beigel, F., Friedrich, M., Probst, C., Sotlar, K., Goke, B., Diegelmann, J. et al. Oncostatin $M$ mediates STAT3-dependent intestinal epithelial restitution via increased cell proliferation, decreased apoptosis and upregulation of SERPIN family members. PLoS ONE 9, e93498 (2014).

61. Kaptein, A., Paillard, V. \& Saunders, M. Dominant negative stat3 mutant inhibits interleukin-6-induced Jak-STAT signal transduction. J. Biol. Chem. 271, 5961-5964 (1996).

62. Wen, Z., Zhong, Z. \& Darnell, J. E. Jr. Maximal activation of transcription by Stat1 and Stat3 requires both tyrosine and serine phosphorylation. Cell 82, 241-250 (1995).

63. Akhtar-Zaidi, B., Cowper-Sal-lari, R., Corradin, O., Saiakhova, A., Bartels, C. F., Balasubramanian, D. et al. Epigenomic enhancer profiling defines a signature of colon cancer. Science 336, 736-739 (2012).

64. Ohbayashi, N., Ikeda, O., Taira, N., Yamamoto, Y., Muromoto, R., Sekine, Y. et al. LIF- and IL-6-induced acetylation of STAT3 at Lys- 685 through PI3K/Akt activation. Biol. Pharm. Bull. 30, 1860-1864 (2007).

65. Tang, Z., Chen, W. Y., Shimada, M., Nguyen, U. T., Kim, J., Sun, X. J. et al. SET1 and p300 act synergistically, through coupled histone modifications, in transcriptional activation by p53. Cell 154, 297-310 (2013).

66. Ali, I., Ruiz, D. G., Ni, Z., Johnson, J. R., Zhang, H., Li, P. C. et al. Crosstalk between RNA Pol II C-terminal domain acetylation and phosphorylation via RPRD proteins. Mol. Cell 74, 1164-74 e4 (2019).

67. Ni, Z., Xu, C., Guo, X., Hunter, G. O., Kuznetsova, O. V., Tempel, W. et al. RPRD1A and RPRD1B are human RNA polymerase II C-terminal domain scaffolds for Ser5 dephosphorylation. Nat. Struct. Mol. Biol. 21, 686-695 (2014).

68. Mei, K., Jin, Z., Ren, F., Wang, Y., Chang, Z. \& Wang, X. Structural basis for the recognition of RNA polymerase II C-terminal domain by CREPT and p15RS. Sci. China Life Sci. 57, 97-106 (2014).

69. Ma, D., Zou, Y., Chu, Y., Liu, Z., Liu, G., Chu, J. et al. A cell-permeable peptide-based PROTAC against the oncoprotein CREPT proficiently inhibits pancreatic cancer. Theranostics 10, 3708-3721 (2020). 